\title{
A Movement-System-Impairment Approach to the Evaluation and Treatment of a Patient with Piriformis Syndrome: A Case Report
}

\author{
Jin-yong Lim, PT, Ph.D. ${ }^{1}$; Il-woo Lee, PT, BS²; Dong-hyun Kim, PT, MS ${ }^{1}$ \\ ${ }^{1}$ Department of Physical Therapy, College of Rehabilitation Sciences, Daegu University, Gyeongsan, South Korea \\ ${ }^{2}$ Department of Physical Therapy, Honsol Hospital, Daegu, South Korea
}

Background Piriformis syndrome (PS) is described as entrapment of the sciatic nerve at the level of the piriformis muscle. Selecting the most effective conservative treatment for PS continues to be a challenge. The movement-system-impairment (MSI) approach is based on the association of symptoms with incorrect movements of the hip. The purpose of this case report was to describe the MSI-based approach to diagnosis in a patient with PS.

Study design Case report.

Observations The patient was a 36-year-old man who presented with sciatic pain in the left lower extremity and anterior hip pain. He began to experience pain approximately 3 months prior to presentation. Prolonged periods spent in a sitting posture aggravated the pain and paresthesia. A clinical examination was performed and the MSI-based diagnosis of hip lateral rotation syndrome was determined. He was instructed to perform specific exercises to address these movement impairments. Exercises were prescribed to address impairments to muscle length, muscle strength, and motor control, all of which are proposed to contribute to the lateral rotation of the hip. The patient reported a reduction in pain and an improvement in his functional activities after 6 weeks of therapy.

Conclusions The present study suggests that the therapeutic application of the MSI approach is effective in patients with PS.

Key words Movement system impairment; Hip lateral rotation syndrome; Sciatic nerve; Piriformis shortness; Piriformis syndrome.

JMST

2018; 2(2): 43-49

Published Online

Dec 31, 2018

pISSN 2635-8573

eISSN 2635-8581

Article History

Received 06 September 2018

Revised 16 October 2018

Accepted 18 October 2018

\section{CONTACT}

isaramjeosaram@gmail.com

Dong-hyun Kim ,

Department of Physical

Therapy, College of

Rehabilitation Sciences,

Daegu University,

Gyeongsan, South Korea

This is an Open-Aceess artiele distributed under the terms of the Creative Commons Attribution Non-Commercial $\mathrm{Li}$ cense (http://creativecommons org/licenses/by-nc/4.0) which permits unrestricted non-commercial use, distribution, and reproduction in any medium provided the original work is properly cited.

\section{INTRODUCTION}

The piriformis muscle originates from the anterior surface of the sacrum and inserts into the upper part of the greater trochanter. ${ }^{1}$ The piriformis muscle is an external rotator, abductor, and flexor of the hip, providing postural stability during standing and walking. ${ }^{2}$

Piriformis syndrome (PS) is a painful musculoskeletal condition, characterized by a combination of symptoms including buttock or hip pain. ${ }^{3}$ The syndrome is caused by sciatic nerve compression due to prolonged or excessive contraction of the piriformis muscle. ${ }^{4}$ PS usually generates pain in the buttock, which may radiate to the lower leg and can be exacerbated when getting up from a sitting position, performing hip adduction and internal rotation, and prolonged periods spent in a sitting position. ${ }^{5}$ In several articles, PS is defined as a peripheral neuritis of the branches of the sciatic nerve caused by an abnormal condition of the piriformis muscle, such as a shortened muscle. ${ }^{6,7}$ The piriformis muscle has a tendency to shorten when the muscle is abnormally stressed. ${ }^{6}$

In addition, repetitive and persistent external hip rotation of the piriformis muscle may increase the stiffness of the posterior hip structures. ${ }^{8}$ Flexion and internal rotation movements of the hip joint are reduced through insufficient posterior gliding of the femur, and restricted hip motion can 
cause compensatory movements of adjacent joints. ${ }^{9}$ The anterior tissues of the femoral head may also cause anterior hip pain due to the tissues impinging during hip flexion., 89

Treatment for PS has traditionally included physical therapy, focused on muscle stretching, extracorporeal shock wave therapy and a combination of local anaesthetic and corticosteroids into the piriformis muscle belly. ${ }^{6,10,11}$ Stretching exercises in particular have been proposed as a mainstay for intervention. ${ }^{12}$ These techniques are generally identified as "piriformis muscle stretching". These techniques seek to emphasize knee and hip flexion with external rotation and adduction of the hip and have been shown effective in stretching of the piriformis muscle. ${ }^{13}$ Gulledge and colleagues (2014) found that the stretching the piriformis muscle over a 5 minute period decreased compression of the sciatic nerve through relaxation of the piriformis muscle by increasing the resting length of the muscle. ${ }^{14}$ However, the physician's diagnoses, which are related to the pathoanatomical source of pain are not designed to guide physical therapists in the management of musculoskeletal disorders. ${ }^{15}$ Sahrmann (2002) has developed a set of diagnoses for the hip, based on movement system impairment (MSI), to guide physical therapy treatment. ${ }^{9}$ The MSI classification is focused on finding functional changes and identifying the direction of the movement causing pain. ${ }^{16}$ It is hypothesized that restoring normal control of movement direction should result in a decrease in pain. To date, no case studies have yet been reported on MSI classification for patients with PS.

The purpose of this case report was to describe the treatment and outcomes of a patient with PS, who was identified as having a hip lateral rotation MSI syndrome.

\section{CASE HISTORY}

The patient was a 36-year-old man who presented with sciatic pain in the left lower extremity and anterior hip pain that had began about 3 months ago. The patient was referred to physical therapy by his physician, with a diagnosis of sciatica. The patient gave informed consent and the rights of the patient were protected. The patient reported that his primary pain began just below his left buttock and went down to his foot. He reported his current buttock pain as 3, while after 5 minutes of walking, the pain was 6 out of 10 using the visual analogue scale $(V A S) .{ }^{17} \mathrm{He}$ stated that his symptoms (pain and paresthesia) were aggravated by working, and other activities and that the symptoms were alleviated by lying supine. He noted that sustained and repeated external rotation of the affected hip increased the symptoms. Prolonged periods spent in a sitting posture also aggravated the symptoms. He also reported occasionally that anterior hip pain occurred during deep squatting. No drugs were used in the periods of exacerbation.

\section{PHYSICAL EXAMINATION}

Movement system examination was performed as described by Sahrmann (2002). ${ }^{9}$ The examination was performed by the first author, a physical therapist with 14 years of experience. The tests were performed by taking the patient through a series of movements in various postures which were analyzed by the examiner. If a particular movement caused pain, a correction was made to the movement in an attempt to reduce symptoms and modify the observed movement impairment.

His preferred standing alignment was a forward sway of pelvic, posterior pelvic tilt, bilateral hip extension and external rotation (Figure 1A). The patient's left foot

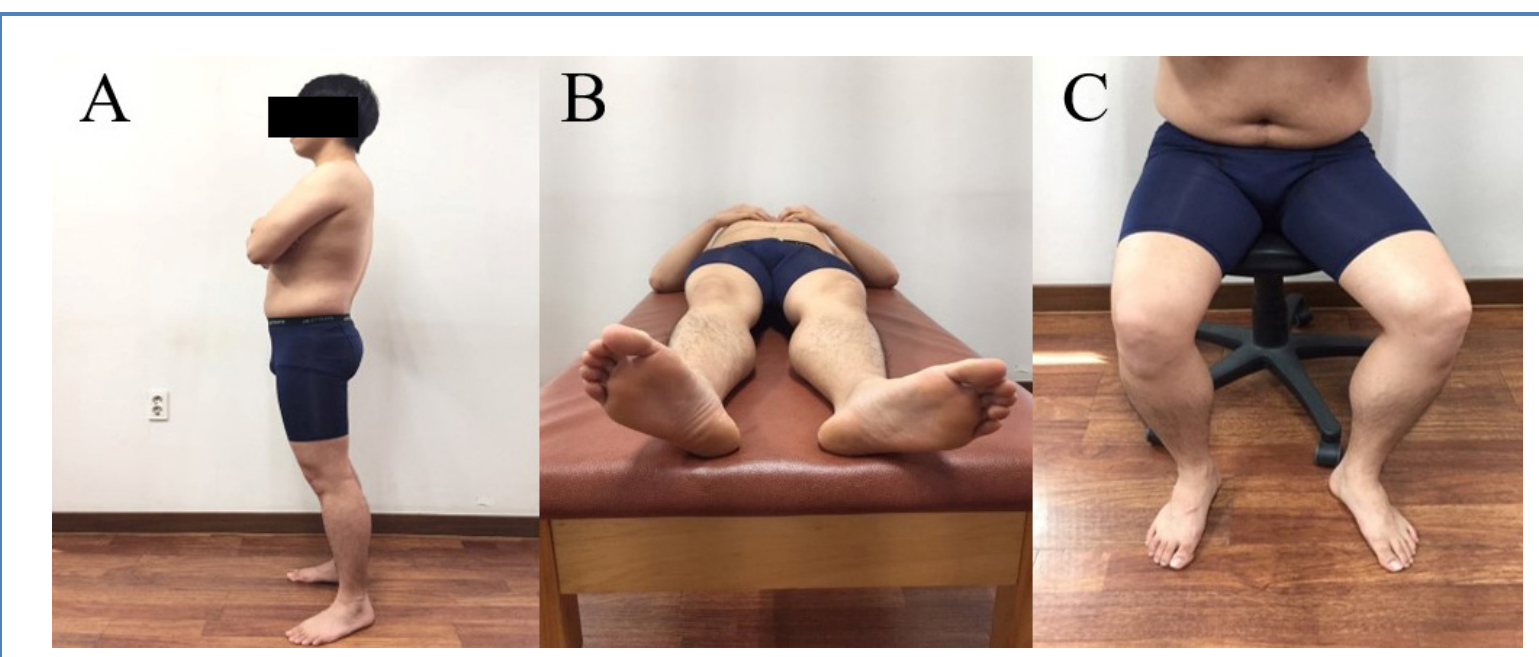

Figure 1. Alignment in different postures. (A) standing position, (B) supine position, (C) sitting position. 
rotated more laterally than the right foot due to the hip external rotation in the supine position (Figure 1B). This alignment occurs when hip external rotators including the piriformis are stiffer than hip internal rotators. ${ }^{9}$ In the sitting position, the left hip showed abduction with external rotation (Figure 1C). The pain was increased during adduction with internal rotation of the hip by the examiner. If the hip was placed in an abduction or an external rotation position, pain was reduced and patient reported being very comfortable.

The patient presented with the following movement impairments. When rocking backwards in a quadruped position, pain was increased in the posterior thigh and inguinal region. The patient was instructed to perform hip abduction with external rotation. The instruction was to increase the flexion range of motion (ROM) of the hip joint by decreasing the stiffness of the piriformis. During reexamination, hip flexion ROM was increased with a reduction in symptoms. While performing an active straight leg raise (ASLR) in the supine position, the hip joint was abducted and externally rotated. At this time, when the hip was placed into the state of adduction and internal rotation by the examiner, the symptoms were increased in the posterior thigh. However, when the hip joint returned to the abducted and externally rotated the symptoms were decreased. The patients' gait, from the mid-stance to the push-off, showed prominent external rotation and extension of the left hip. These movements were more obvious to the observer when the patient walked rapidly.

Manual muscle testing was performed according to Kendall (2005) and results revealed weakness of the left hip flexors and internal rotators compared to the right (Table 1). ${ }^{18}$ A positive FADIR (hip joint flexion, adduction and internal rotation) test indicates anterior impingement of the femoral head against the acetabulum. ${ }^{19}$ This test was performed by an examiner who passively moved the hip of the patient into approximately $90^{\circ}$ of flexion, $10^{\circ}$ of adduction, and $10^{\circ}$ of internal rotation in a supine position. The pain experienced by the patient was increased during this test (Table 2). The FAIR (hip joint flexion, adduction and internal rotation) test is a sensitive and specific test for detection of irritation of the sciatic nerve by the piriformis. ${ }^{20}$ The FAIR test was performed with the patient lying on his side, with the affected side up, the hip flexed to an angle of $90^{\circ}$ and the knee flexed to an angle of $60^{\circ}$. While stabilizing the pelvis, the examiner adducted and internally rotated the hip by applying downward pressure to the knee. ${ }^{21}$ The FAIR test was painful to the patient (Table 2 ). The length tests of the hip external rotators were performed using the prone hip internal rotation ROM test. ${ }^{22}$ The hip internal rotation ROM was smaller at the left side than the right side (Table 2). On palpation there was tenderness and the patients' symptoms could be reproduced by applying pressure to the belly of the left piriformis muscle in the gluteal region.

\section{DIAGNOSIS}

Considering the results of the above findings, the patients' MSI diagnosis is hip lateral rotation syndrome (Table 3). ${ }^{9}$ As the results of the patient's examination were consistent with this MSI syndromes, the MSI approach was indicated for his treatment.

\section{TREATMENT}

Based on the MSI diagnosis, physical therapy was recommended at a frequency of three times per week for a duration of up to 6 weeks. The patient was taught to avoid positions and movements that promoted excessive lateral rotation of the hip. He was then taught exercises that were designed to address specific movement impairments, muscle weakness and length identified during the exam (Table 4). During the first week of treatment, gentle myofascial release was also performed on the piriformis for 10 minutes, over a course of three treatments.

Table 1. Manual muscle test: comparison over 6 weeks

\begin{tabular}{ccccc}
\hline Characteristics & Initial : right & Initial : left & 6 weeks right & 6 weeks left \\
\hline Hip internal rotation & $3+/ 5$ & $2+/ 5$ & $4+/ 5$ & $4+/ 5$ \\
Hip abduction & $4 / 5$ & $4-/ 5$ & $4+/ 5$ & $4+/ 5$ \\
Hip flexion & $3 / 5$ & $2+/ 5$ & $3+/ 5$ & $3+/ 5$ \\
Hip lateral rotation & $4+/ 5$ & $4-/ 5$ (discomfort) & $4+/ 5$ & $5 / 5$ \\
\hline
\end{tabular}

$5 / 5$, normal strength; $4+/ 5$, able to hold against moderate to strong resistance; $4 / 5$, able to hold against moderate resistance; $4-/ 5$, able to hold against slight to moderate resistance; $3+/ 5$, able to hold against minimal resistance; $3 / 5$, able to hold against gravity but not against additional minimal resistance applied manually; $2+/ 5$, moves through partial range of motion against gravity. 
Table 2. Outcomes of other measures

\begin{tabular}{ccc}
\hline Measure & Initial & 6 weeks \\
\hline VAS & At rest $: 3 / 10$ & At rest $: 0 / 10$ \\
With activity : $6 / 10$ & With activity : $0 / 10$
\end{tabular}

Table 3. Movement system impairment diagnosis

\begin{tabular}{cccccc}
\hline Diagnosis & Movement impairment & Posture & Muscle strength & Muscle length & Pain \\
\hline \multirow{2}{*}{$\begin{array}{c}\text { Hip lateral } \\
\text { rotation syndrome }\end{array}$} & Hip LR during walking. & $\begin{array}{c}\text { Swaback posture } \\
\text { Hip LR during ASLR }\end{array}$ & $\begin{array}{c}\text { Heak left iliopsoas } \\
\text { Hip LR }\end{array}$ & Weak hip internal \\
& & Pelvic posterior tilt & rotators & Short left Piriformis & With left hip IR and \\
adduction
\end{tabular}

Table 4. Intervention: exercises

\begin{tabular}{|c|c|c|c|c|}
\hline Exercise & Instructions & Purpose & Frequency & Repetitions \\
\hline $\begin{array}{l}\text { Quadruped rock } \\
\text { back }\end{array}$ & $\begin{array}{l}\text { On hands and knees, pull in abs, } \\
\text { rock back toward heels. }\end{array}$ & $\begin{array}{l}\text { Improve hip flexion } \\
\text { Elongate short } \\
\text { piriformis }\end{array}$ & $\begin{array}{l}3 \text { times per } \\
\text { week }\end{array}$ & $\begin{array}{l}15 \text { times, hold for } 10 \sim 15 \\
\text { seconds at end } \\
2 \text { set }\end{array}$ \\
\hline $\begin{array}{l}\text { Side-lying hip IR } \\
\text { with sandback ( } 3 \mathrm{~kg})\end{array}$ & $\begin{array}{l}\text { Pull in abs. } \\
\text { Move in hip joint only } \\
\text { Do not let pelvis rotate } \\
\text { Alternately for both side }\end{array}$ & $\begin{array}{l}\text { Strengthen hip internal } \\
\text { rotators, Elongate short } \\
\text { piriformis }\end{array}$ & $\begin{array}{l}3 \text { times per } \\
\text { week }\end{array}$ & $\begin{array}{l}15 \text { times, hold for } 10 \sim 15 \\
\text { seconds at end Start without } \\
\text { weight and build gradually } \\
\text { weight } \\
2 \text { set }\end{array}$ \\
\hline Prone hip IR & $\begin{array}{l}\text { Pull in abs. } \\
\text { Do not let pelvis rotation } \\
\text { Alternately for both side }\end{array}$ & $\begin{array}{c}\text { Elongate short } \\
\text { piriformis }\end{array}$ & $\begin{array}{l}3 \text { times per } \\
\text { week }\end{array}$ & $\begin{array}{l}15 \text { times, hold for } 10 \sim 15 \\
\text { seconds at end } \\
1 \text { set }\end{array}$ \\
\hline Sitting hip flexion & $\begin{array}{l}\text { Pull in abs. } \\
\text { Do not let pelvis posterior rotate } \\
\text { Alternately for both side }\end{array}$ & Strengthen hip flexors & $\begin{array}{l}3 \text { times per } \\
\text { week }\end{array}$ & $\begin{array}{l}15 \text { times, hold for } 10 \sim 15 \\
\text { seconds at end } \\
\text { As tolerated, } \\
\text { gradually increase ROM } \\
2 \text { set }\end{array}$ \\
\hline
\end{tabular}

The goals for the 6-month treatment were the following:

First, we attempted to stretch the piriformis muscle to reduce the pressure on the sciatic nerve. Then, we aimed to stretch the muscle to the point where symptoms no longer occurred to prevent excessive nerve compression. Second, we tried to make the patient able to sit on a chair for at least an hour, walk with decreased pain or numbness and recover functional activities. 


\section{OUTCOME}

\section{6 weeks re-assessment}

At the 6 weeks re-assessment the patient stated that he had been gradually improving and the pain was generally decreased. The VAS score was 0 out of 10 on resting, meaning that the symptoms were improved by 3 points. During gait, the symptoms in the lower extremity were significantly improved, with the VAS score from decreasing from 6 out of 10 to 0 out of 10 . The pain in the gluteal region during rolling in the supine position was removed. The patient reported no further gluteal pain or paresthesia whilst sitting for 1 hour. He also said that the pain no longer increased during work. No anterior hip pain was observed during a deep squat exercise. The patient stated that the left foot was no longer rotated laterally in the supine position and the left thigh was also abducted and externally rotated in the sitting position. The patient could perform ASLR without hip external rotation. During gait, it was observed that the amount of hip external rotation and extension was decreased, although not completely. The muscle length test, muscle strength test, special test and performances for the patient were reexamined, and they showed significant improvements (Tables 1 and 2). The patient exercised for about 50 minutes per a visit and he returned to visit the therapist 18 times for 6 weeks.

\section{DISCUSSION}

We have reported on the examination, diagnosis, and treatment of an individual with PS. Standardized examination including alignment and movement impairment was performed and an MSI diagnosis was determined. The symptoms observed in the patient were consistent with the hip lateral rotation syndrome from the MSI diagnosis. Although the patient's symptoms have improved due to prescribed exercise, we recognize that other therapeutic approaches can achieve similar results.

After 6 weeks, the patient reported the decreased hip pain and thigh numbness during sitting and gait. Backward rocking in the quadruped position and prone hip internal rotation exercises were performed to stretch the piriformis muscle for 6 weeks. As a result, the prone hip internal rotation ROM was increased. Gajdosik (2001) reported that muscle stretching improves the muscle expansibility, reduces muscle stiffness, and is effective to increase the joint ROM by adding sarcomeres into the muscles. ${ }^{23}$ It is believed that the exercises contributed to reduce the pressure on the sciatic nerve by stretching the piriformis muscle. In particular, the pain that the patient experienced during rolling movements on a bed was reduced after 6 weeks of exercise. It is believed that improved hip ROM, caused by the increased length of the piriformis muscle influenced the improvement of the hip function. In addition, exercise involving backward rocking in a quadruped position expands the posterior capsule of the hip and allows posterior gliding of the femoral head. ${ }^{9}$ It is believed that the posterior gliding of the femoral head might have helped to decrease the anterior hip pain experienced by the patient during a deep squat.

The patient reported severe muscle weakness in the internal rotators of the hip. Internal hip rotation exercises were performed with the patient lying on his side to strengthen weakened muscles. The purpose of the exercise was to improve the strength of the anterior fibers of the gluteus medius and minimus as internal rotators of the hip. After 6 weeks, the strength grades of hip internal rotators were $4+/ 5$, as defined by Kendall et al (2005). ${ }^{18}$ The patient increased the strength of the hip internal rotators as well as lengthening the hip external rotators by following the exercise regime. We believe that the increased strength of the hip internal rotators could increase the stiffness of them. It is considered that this increased stiffness could move the left foot into a neutral position, preventing external rotation of the hip during ASLR and help the hip joint to align normally in supine and sitting positions.

The patient had a typical Swayback posture, which resulted in stretch weakness of the iliopsoas muscles. ${ }^{18}$ The sitting hip flexion exercise was performed to strengthen the weakened iliopsoas muscles as flexors of the hip. After 6 weeks, the strength grade of the iliopsoas muscles had increased from $2+/ 5$ to $3+/ 5$. In theory, a muscle with a strength grade of $3+/ 5$ should be able to hold against minimal resistance. Unfortunately, the excessive extension ROM of the hip was not corrected, possibly because the increased iliopsoas strength was not enough to correct it.

This study has several limitations. First, the only one patient participated in this study, so it is difficult to generalize the results. Second, there was no follow-up study, so there can be no suggestion of sustainability of the result. Future studies are required, using a larger sample size. These studies should be designed to determine the long-term follow-up effectiveness of the MSI approach in the management of subjects with PS.

\section{CONCLUSIONS}

We described the evaluation and treatment of a patient 
with PS. The patient also was taught an exercise program designed to address muscle and movement impairments that were thought to contribute to the MSI diagnosis of hip lateral rotation. The patient reported a reduction of pain and an improvement in his functional activities at 6 weeks. The present study suggests that the therapeutic application of the MSI approach is effective in patients with PS.

\section{Key Points}

Question Is the MSI-based approach effective for a patient with PS?

Findings We found that application of MSI techniques improves pain and movement in a patient with PS.

Meaning We suggest that the MSI-based approach can be used in clinical settings for patients with PS.

\section{Article information}

Conflict of Interest Disclosures: None.

Funding/Support: None.

Acknowledgment: None.

\section{REFERENCES}

1. Meknas K, Johansen O, Kartus J. Retro-trochanteric sciatica-like pain: Current concept. Knee Surgery, Sports Traumatology, Arthroscopy. 2011;19(11):1971.

2. Norbury JW, Morris J, Warren KM, et al. Diagnosis and management of piriformis syndrome. Pract NEurol. 2012;12(6):24-27.

3. Cramp F, Bottrell O, Campbell H, Ellyatt P, SmithC, Wilde B. Non-surgical management of piriformis syndrome: A systematic review. Physical Therapy Reviews. 2007;12(1):66-72.

4. Jankovic D, Peng P, van Zundert A. Brief review: Piriformis syndrome: Etiology, diagnosis, and management. Canadian Journal of Anesthesia/Journal can Adien D'anesthésie. 2013;60(10):1003-1012.

5. Kirschner JS, Foye PM, Cole JL. Piriformis syndrome, diagnosis and treatment. Muscle \& Nerve. 2009;40(1): 10-18.

6. Mondal M, Sarkar B, Alam S, et al. Prevalence ofpiriformis tightness in healthy sedentary individuals:A cross-sectional study. IJHSR. 2017;7(7):134-142.

7. Tonley JC, Yun SM, Kochevar RJ, Dye JA, Farrokhi S, Powers CM. Treatment of an individual with piriformis syndrome focusing on hip muscle strengthening and movement reeducation: A case report. Journal of Orthopaedic \& Sports Physical Therapy. 2010;40(2):
103-111.

8. Lee D, Vleeming A. An integrated therapeutic approach to the treatment of pelvic girdle pain. Movement, Stability \& Lumbopelvic Pain (Second Edition): Elsevier; 2007:621-638.

9. Sahrmann S. Diagnosis and treatment of movement impairment syndromes. Elsevier Health Sciences; 2002.

10. Dezawa A, Kusano S, Miki H. Arthroscopic releaseof the piriformis muscle under local anesthesia for piriformis syndrome. Arthroscopy. 2003;19(5):554-557.

11. Huang F, Chen X, Mu J-p. Clinical study on extracorporeal shock wave therapy plus electroacupuncture for myofascial pain syndrome. Journal of Acupuncture and Tuina Science. 2014;12(1):55-59.

12. Papadopoulos EC, Khan SN. Piriformis syndrome and low back pain: A new classification and review of the literature. The Orthopedic Clinics of North America. 2004;35(1):65-71.

13. Meknas K, Kartus J, Letto JI, Flaten M, Johansen O. A 5 -year prospective study of non-surgical treatment of retro-trochanteric pain. Knee Surgery, Sports Traumatology, Arthroscopy. 2009;17(8):996-1002.

14. Gulledge BM, Marcellin-Little DJ, Levine D, et al. Comparison of two stretching methods and optimization of stretching protocol for the piriformis muscle. Medical Engineering \& Physics. 2014;36(2):212-218.

15. Caldwell C, Sahrmann S, Van Dillen L. Use of a movement system impairment diagnosis for physical therapy in the management of a patient with shoulder pain. Journal of Orthopaedic \& Sports Physical Therapy. 2007;37(9):551-563.

16. Harris-Hayes M, Van Dillen LR, Sahrmann SA. Classification, treatment and outcomes of a patient with lumbar extension syndrome. Physiotherapy Theory and Practice. 2005;21(3):181-196.

17. Price DD, Bush FM, Long S, Harkins SW. A comparison of pain measurement characteristics of mechanical visual analogue and simple numerical rating scales. Pain. 1994;56(2):217-226.

18. Kendall FP, McCreary EK, Provance PG, Rodgers M, Romani WA. Muscles: Testing and function, with posture and pain (kendall, muscles). Philadelphia: Lippincott Williams \& Wilkins; 2005.

19. Kuhlman GS, Domb BG. Hip impingement: Identifying and treating a common cause of hip pain. American Family Physician. 2009;80(12):1429-1434.

20. Fishman LM, Dombi GW, Michaelsen C, et al. Piriformis syndrome: Diagnosis, treatment, and outcome-a 10-year study. Archives of Physical Medicine and Rehabilitation. 2002;83(3):295-301. 
21. Hashemirad F, Karimi N, Keshavarz R. The effect of Kinesio taping technique on trigger points of the piriformis muscle. Journal of Bodywork and Movement Therapies. 2016;20(4):807-814.

22. McGovern RP, Kivlan BR, Martin RL. Length change of the short external rotators of the hip in common stretch positions: A cadaveric study. International Journal of Sports Physical Therapy. 2017;12(7):1068.

23. Gajdosik RL. Passive extensibility of skeletal muscle: Review of the literature with clinical implications. Clinical Biomechanics. 2001;16(2):87-101. 